\title{
Changed immune and miRNA response in RAW264.7 cells infected with cell wall deficient mycobacterium tuberculosis
}

\author{
ZHENGJUN YI $^{1 *}, \mathrm{KUNSHAN} \mathrm{GAO}^{1 *},{\text { RUIFANG } \mathrm{LI}^{2} \text { and YURONG FU }}^{1,2}$ \\ ${ }^{1}$ Department of Laboratory Medicine, Key Laboratory of Clinical Laboratory Diagnostics in Universities of \\ Shandong and Medical Priority Speciality of Clinical Laboratory in Shandong Province, Weifang Medical University; \\ ${ }^{2}$ Department of Medical Microbiology, Weifang Medical University, Weifang, Shandong 261053, P.R. China
}

Received November 3, 2016; Accepted January 31, 2018

DOI: $10.3892 / \mathrm{ijmm} .2018 .3471$

\begin{abstract}
Cell wall deficient (CWD) forms of Mycobacterium tuberculosis (Mtb) confers a marked resistance to immune system of the host. However, there is limit data on the effect of intracellular CWD-Mtb infection on macrophages. In the study, effects of CWD-Mtb on cell viability, cytokine response and miRNA expression of macrophages were analyzed. Cell viability was reduced, levels of interleukin-1 $\alpha$ (IL-1 $\alpha)$, IL-1 $\beta$, IL-6, IL-10 and interferon- $\gamma($ IFN- $\gamma)$ were also significantly changed after infection of RAW264.7 cells with CWD-Mtb. A total of 105 miRNAs were deregulated between CWD-Mtb and wild Mtb group, and among them, miR-29b was upregulated in CWD-Mtb group. Downregulation of miR-29b resulted in significant elevation level of IFN- $\gamma$ mRNA. Involved signaling pathways of potential target genes of differentially expressed miRNAs mainly focused on $\mathrm{T}$ cell receptor signaling pathway, MAPK signaling pathway, neurotrophin signaling pathway, and regulation of actin cytoskeleton. Taken together, the results showed that cytokine production of CWD-Mtb infected macrophages was altered and many miRNAs were involved in regulation of macrophage response to CWD-Mtb infection, which probably determined the differential outcome following different phenotype Mtb infection. These findings open up a new and interesting avenue for an improved understanding of pathogenesis of CWD-Mtb.
\end{abstract}

Correspondence to: Dr Yurong Fu, Department of Laboratory Medicine, Key Laboratory of Clinical Laboratory Diagnostics in Universities of Shandong and Medical Priority Speciality of Clinical Laboratory in Shandong Province, Weifang Medical University, 7166 Baotong Xi (Western) Street, Weicheng, Weifang, Shandong 261053, P.R. China

E-mail: yifuyurong@163.com

${ }^{*}$ Contributed equally

Key words: mycobacterium tuberculosis, cell wall deficient, miRNA, macrophage, immune response

\section{Introduction}

Cell wall deficient (CWD or L) forms of Mycobacterium tuberculosis (Mtb), characterized by loss of cell wall components, are usually considered as a product of interaction between wild Mtb and defence system of host. As a result, CWD-forms of Mtb confers a marked resistance of mycobacteria to immune system of host and antibiotics (1). CWD-forms of Mtb have been found in specimens from $42 \%$ patients with freshly or previously diagnosed pulmonary $\mathrm{TB}$, and of all bacteria, CWD-forms of Mtb predominate in vivo (2). CWD-forms of Mtb are responsible for chronic and persistent tuberculosis (TB) infection that constitutes one of the major hurdles in control of TB $(3,4)$. Unfortunately, over the last several decades, investigations on CWD-Mtb have been ignored and overlooked. Recently, interest in CWD-Mtb has been reborn due to its significant role in chronic and persistent TB disease. Despite long history of TB research, underlying pathogenic mechanism by which CWD-forms of Mtb counteract the bactericidal activities and survive in host cells still remains obscure. Macrophages, the main targets of mycobacterial infection, play an important role in the pathogenesis of $\mathrm{Mtb}$ infection. Different Mtb strains could induce macrophage to produce distinct responses $(5,6)$. Many studies have explored the interaction between macrophages and Mtb with cell wall (wild Mtb) (7-9). However, there is limited data on the effect of intracellular CWD-Mtb infection on macrophages.

MicroRNAs (miRNAs) are crucial biological regulators that bind complementarily to 3 '-untranslated regions (3'-UTRs) of target mRNAs leading to mRNA cleavage or translational inhibition (10). The emerging roles of miRNAs in regulating immune responses have attracted increasing attention in recent years and miRNAs play a major role in the prognosis of infectious diseases $(11,12)$. Many studies have shown that regulation of specific miRNAs during mycobacterial infection can either enhance immune response or facilitate pathogen immune evasion. For instance, miRNA-21 could impair anti-mycobacterial responses by targeting interleukin-12 (IL-12) and Bcl-2 (13); miR-124 played a negative regulatory role in fine-tuning inflammatory response in macrophages upon mycobacterial infection (14); miR-223 was critical for the control of TB by regulating leukocyte chemotaxis (15); miR-155 could 
enhance survival of Mtb in macrophages (16). These results suggest that miRNAs play important roles in TB infectious disease. However, the immunoregulatory role of miRNAs in CWD-Mtb infection is still poorly understood.

The present study was carried out to determine the effects of intracellular CWD-Mtb infection on cell viability and immune response of macrophages, and further elucidate the potential role of miRNA in macrophage response to CWD-Mtb infection.

\section{Materials and methods}

Preparation of CWD-Mtb. Mtb strain H37Rv (wild Mtb with cell wall) was grown in Middlebrook 7H9 broth supplemented with 10\% OADC enrichment (BD Biosciences, San Jose, CA, USA), $0.5 \%$ glycerol and $0.05 \%$ Tween- 80 . Wild Mtb was used to prepare CWD-forms of Mtb by nutrient starvation method as described by Markova et al (1). Mid-log phase suspension of H37Rv culture was pelleted, resuspended in DMEM medium plus 10\% fetal bovine serum (FBS) and HEPES, vortexed for $2 \mathrm{~min}$ and sonicated for $5 \mathrm{~min}$. Following sonication, bacteria were dispersed by aspiration with needle. Dispersed bacterial suspension was then allowed to stand in a vertical position for 5 min and top portion of suspension was used for subsequent infection experiment. CWD-Mtb was examined and confirmed by Ziehl-Neelsen staining and electron microscopy.

Cell culture and infection. One day prior to infection, RAW264.7 cells were cultured in DMEM medium supplemented with $10 \%$ heat-inactivated FBS (complete medium) under standard conditions $\left(37^{\circ} \mathrm{C}\right.$ and $5 \% \mathrm{CO}_{2}$ humidity). Twenty-four hours later, cells at $\sim 80 \%$ confluence were infected with either CWD-Mtb or wild Mtb at a multiplicity of infection (MOI) of 10 bacteria/cell. At $4 \mathrm{~h}$ post-infection, cells were carefully washed 3 times with fresh DMEM medium to remove extracellular bacteria and further cultured in prewarmed complete medium for subsequent analysis.

Methyl thiazolyl tetrazolium (MTT) assay and intracellular bacterial growth. RAW264.7 cells were infected with either CWD-Mtb or wild Mtb as mentioned above. Effect of intracellular Mtb infection on cell viability was determined by MTT assay. Briefly, culture medium was discarded at 4,12 or $24 \mathrm{~h}$ post infection and cells were then incubated with MTT solution for $2 \mathrm{~h}$. Precipitates were then dissolved with dimethyl sulfoxide and optical density (OD) at $570 \mathrm{~nm}$ was measured using a microplate reader. Data were collected in triplicate for each sample. Infected cells from at least three parallel wells were harvested and lysed by adding $1 \%$ Triton X-100 at $24 \mathrm{~h}$ time-point. Cell lysates were serially diluted and seeded on Middlebrook 7H10 solid plates for wild Mtb growth and Middlebrook 7H9 semisolid plates (both from BD Difco, Franklin Lakes, NJ, USA) for CWD-Mtb growth in, at least, duplicates. Colony forming units (CFUs) were enumerated after 30 days for wild Mtb and 5 days for CWD-Mtb, respectively. Three separate biological replicates were performed.

Cytokine analysis. Culture supernatants were collected from either wild Mtb or CWD-Mtb infected cells at $24 \mathrm{~h}$ post-infection and immediately stored in liquid nitrogen. Levels of
IL-1 $\alpha$, IL-1 $\beta$, IL-6, IL-10, TNF- $\alpha$ and interferon- $\gamma$ (IFN- $\gamma)$ in culture supernatants were measured using commercially available enzyme-linked immunosorbent assay (ELISA) kits (BioSource, Nivelles, Belgium) according to the manufacturer's instructions. For each group, three separate biological replicates were performed. All culture supernatants were measured in triplicate (technical replicates) and results from technical replicates were combined.

miRNA expression and data processing. Cells were harvested at $24 \mathrm{~h}$ post-infection and stored in liquid nitrogen until RNA was extracted. Total RNA was isolated from harvested cells using TRIzol reagent (Invitrogen, Carlsbad, CA, USA) and further purified with a miRNeasy mini kit (Qiagen, Hilden, Germany) following the manufacturer's instructions. RNA quantity and quality were assessed using a NanoDrop Spectrophotometer (NanoDrop Technologies, Wilmington, DE, USA) and electrophoresis. After having passed RNA quantity and quality measurement, each sample was labeled using miRCURY ${ }^{\mathrm{TM}} \mathrm{Hy}^{\mathrm{TM}}$ Power labeling kit and hybridized on mouse miRCURY'TM LNA array (v.16.0) (Exiqon, Vedbaek, Denmark) according to the manufacturer's protocol. Three arrays were used for each group. Following hybridization, microarray slides were washed and scanned using an Axon GenePix 4000B microarray scanner (Axon Instruments, Foster City, CA, USA). GenePix Pro 6.0 software (Axon Instruments) was used to analyze acquired array images. After quantile normalization of raw data, fold change filtering method was used to select miRNA with at least a 2 -fold change for further statistical analysis. U6 (U6 small nuclear RNA) was used as an internal control. Statistical significance was determined by false discovery rate (FDR) $<0.05$ to identify differentially expressed miRNAs. Hierarchical clustering was performed based on Pearson's correlation for differentially expressed miRNAs.

$R T$-qPCR analysis. Some deregulated miRNAs were randomly selected from among those showing the greatest fold changes for confirmation by real-time reverse transcription quantitative PCR (RT-qPCR). Total RNA (200 ng for each sample) was used for the synthesis of first stranded cDNA in $20 \mu 1 \mathrm{RT}$ reaction containing $15 \mathrm{nM}$ miRNA-specific primers (available on request), 1X RT buffer, $0.25 \mathrm{mM}$ each of dNTPs, $2 \mathrm{U} / \mu \mathrm{l}$ reverse transcriptase and $0.6 \mathrm{U} / \mu 1$ RNase inhibitor. Obtained $2.5 \mathrm{ng}$ cDNA for each sample was then used for PCR analysis using a GeneAmp PCR system 9700 (Applied Biosystems, Perkin-Elmer, Foster City, CA, USA) in $10 \mu 1$ reaction including $1 \mathrm{X}$ master mix and $0.5 \mu \mathrm{M}$ each primer. Normalized, relative gene expression was calculated using standard $2^{-\Delta \Delta \mathrm{Ct}}$ method. Data are presented as fold change in gene expression normalized to level of internal control U6. Each qPCR reaction was run three independent times, with technical triplicates in each reaction.

Correlation between miR-29b level and IFN- $\gamma$ expression in CWD-Mtb infected RAW264.7 cells. miR-29b mimics, miR-29b inhibitors and nonsense RNA controls were purchased from GenePharma (Shanghai, China). Briefly, RAW264.7 cells were seeded in triplicate in 24-well plates at a concentration of $1 \times 10^{5}$ cells/well in $100 \mu 1$ complete medium. 


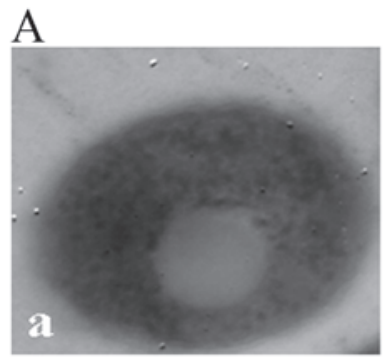

B

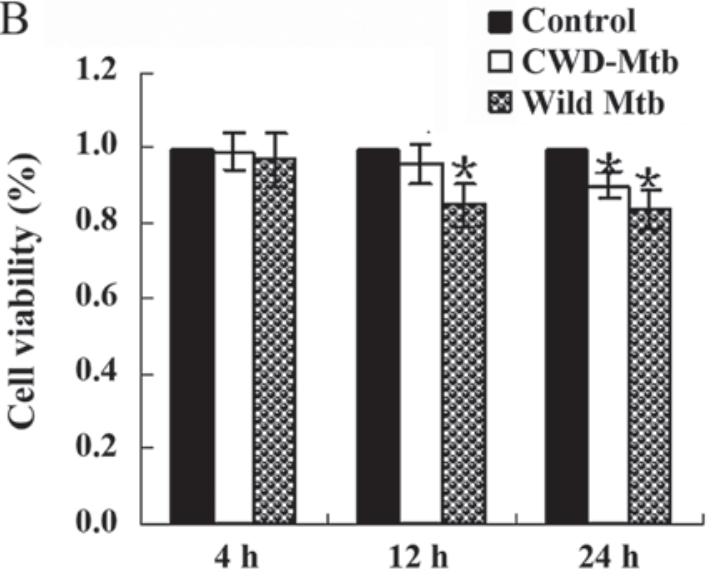

Figure 1. Electron microscopy images and methyl thiazolyl tetrazolium (MTT) assay. (A) Transmission electron microscopy of ultrathin sections of Mycobacterium tuberculosis (Mtb) H37Rv with (a) cell wall and (b) cell wall deficient (CWD)-Mtb. CWD-Mtb was covered only by a 'unit' cytoplasmic membrane. Bars, $400.00 \mathrm{~nm}$. (B) Measurement of cell viability of either CWD-Mtb or wild Mtb infected as well as non-infected RAW264.7 cells using MTT assay. Results are shown as means \pm SD of 3 individual experiments. ${ }^{*} \mathrm{P}<0.05$ vs. non-infected control.

At $4 \mathrm{~h}$ post-infection with CWD-Mtb, culture medium was removed and cells were then transiently transfected with miR-29b mimics, miR-29b inhibitors or nonsense RNA controls in equimolar amounts with HiPerFect transfection reagent (Qiagen) according to the manufacturer's protocols. Cells transfected with only transfection reagent were used as mock control. Culture medium was replaced with fresh complete DMEM medium at $6 \mathrm{~h}$ post-transfection and cells were then harvested after transfection for $24 \mathrm{~h}$. RT-qPCR was used to determine IFN- $\gamma$ mRNA expression. Each experiment was repeated three separate times.

Kyoto encyclopedia of genes and genomes pathway analysis based on miRNA expression profile. Target genes of deregulated miRNAs were predicted by TargetScan (http://www. targetscan.org/) and pathway analysis was used to find significant pathways of differential genes according to Kyoto encyclopedia of genes and genomes (KEGG).

Statistical analysis. Data are presented as means \pm standard deviation (SD) of three independent experiments. One-way ANOVA analysis or t-test was used for statistical analysis. $\mathrm{P}<0.05$ was considered statistically significant.

\section{Results}

Cell viability and intracellular bacterial growth. CWD-forms of Mtb were induced from wild Mtb by nutrient starvation

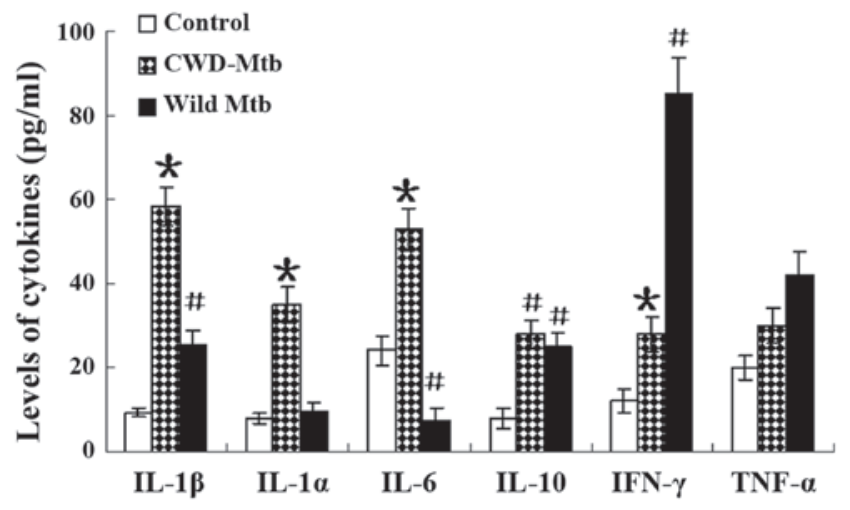

Figure 2. Determination of cytokines production with enzyme-linked immunosorbent assay (ELISA). Culture supernatants of RAW264.7 cells were harvested after $24 \mathrm{~h}$ post-infection. Levels of cytokines in culture supernatants were determined by ELISA. Data were presented as means \pm SD from three independent experiments. ${ }^{*} \mathrm{P}<0.05$ compared with wild Mycobacterium tuberculosis (Mtb) group as well as non-infected control; ${ }^{\#} \mathrm{P}<0.05$ compared with non-infected control.

stress, which showed non-acid-fast staining under light microscopy and was bound only by a single unit membrane under electron microscopy (Fig. 1A). Effect of CWD-Mtb on cell viability during intracellular infection of macrophage was detected by MTT assay. As shown in Fig. 1B, compared with non-infected cell controls, cell viability of wild Mtb infected cells was significantly reduced at 12 and $24 \mathrm{~h}$ post-infection, while cell viability of CWD-Mtb infected cells was obviously decreased only at $24 \mathrm{~h}$ post-infection. However, there was no significant difference between wild Mtb group and CWD-Mtb group at 12 or $24 \mathrm{~h}$ post-infection. Data also showed that there was no significant difference in intracellular bacterial growth between CWD-Mtb group and normal Mtb group at $24 \mathrm{~h}$ post-infection (data not shown).

Measurement of cytokine production. Cytokines have a critical role in regulating innate and adaptive immunity and changes in the pattern of cytokine production clearly contribute to TB progression (17,18). Different mycobacteria strains could induce macrophages to produce distinct pattern of cytokines (19). To study cytokine response of macrophages to intracellular CWD-Mtb infection, levels of IL-1 $\alpha$, IL-1 $\beta$, IL-6, IL-10, TNF- $\alpha$ and IFN- $\gamma$ in supernatants of infected cells were evaluated by ELISA. Levels of IL-1 $\alpha$, IL-1 $\beta$, IL- 6 and IFN- $\gamma$ were changed in CWD-Mtb group compared with wild Mtb group as well as non-infected control; IL-10 was only altered in CWD-Mtb group compared with wild Mtb group. However, there was no obvious difference for TNF- $\alpha$ level between the three groups (Fig. 2).

miRNA expression. Roles of miRNA have been highlighted in TB infection recently (13-16). To investigate whether miRNAs were involved in the response of macrophages to intracellular CWD-Mtb infection, total RNA was isolated from infected cells and miRNA expression was determined. Compared to control, downregulated 43 miRNAs and upregulated 28 miRNAs were observed in both CWD-Mtb group and wild Mtb group, simultaneously (Fig. 3A). A total of 105 miRNAs were deregulated between CWD-Mtb group and wild Mtb 

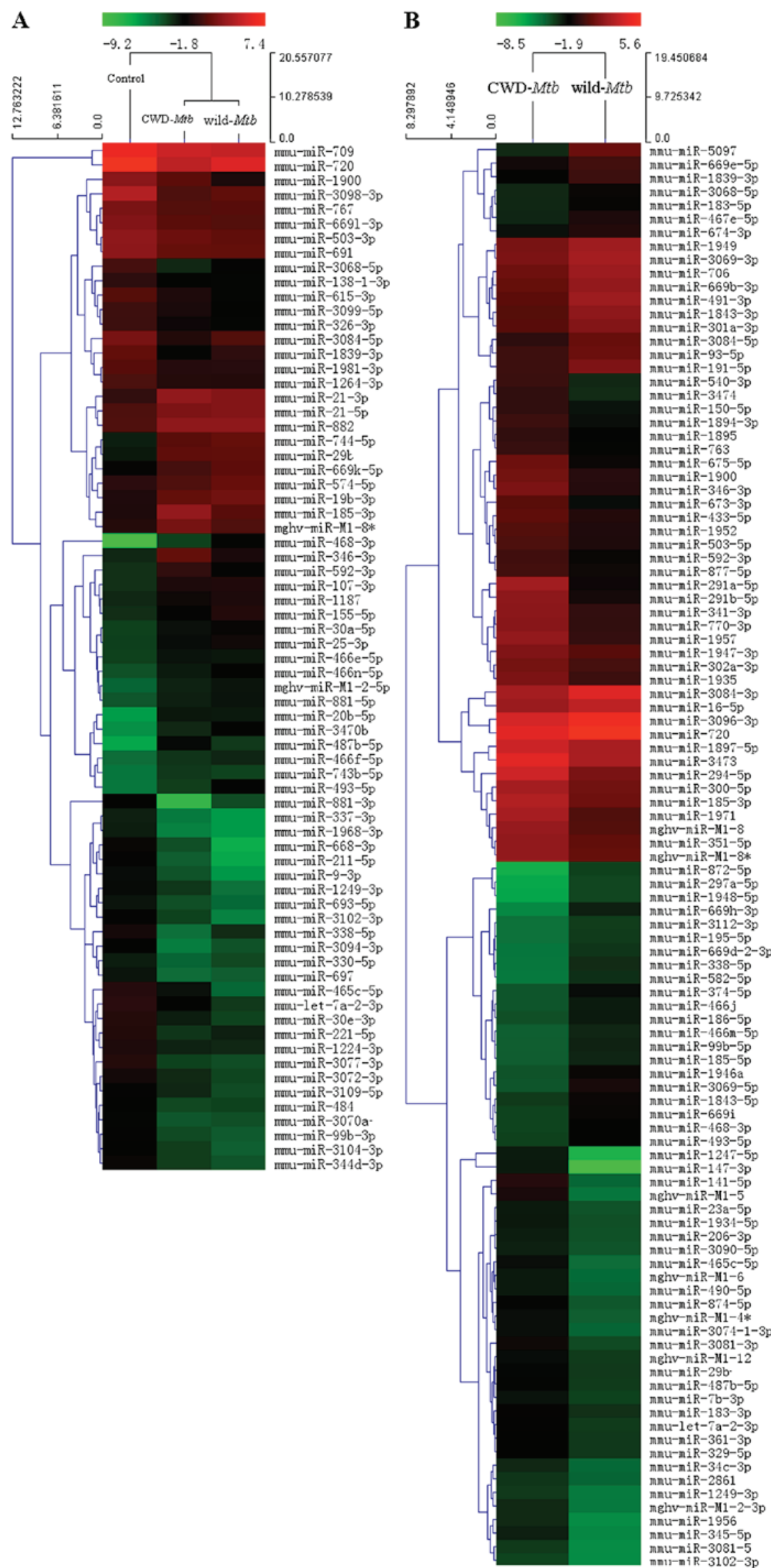

Figure 3. (A and B) Hierarchical clustering of differentially expressed miRNAs between cell wall deficient (CWD)-Mycobacterium tuberculosis (Mtb) group and wild Mtb group. Red indicates high relative expression, and green indicates low relative expression. Microarray results were obtained from three independent samples. These miRNAs were significantly differentially expressed (fold $>2$, FDR $<0.05$ ). 


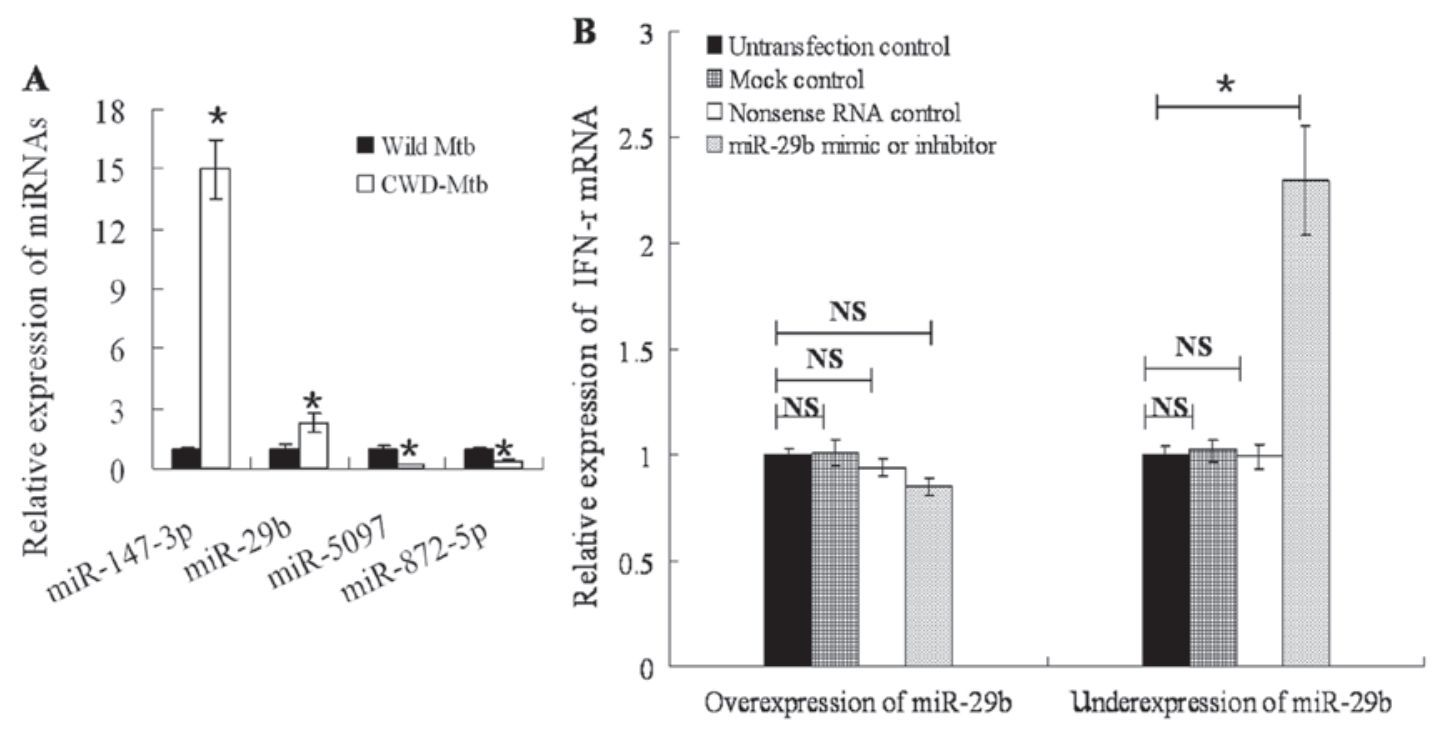

Figure 4. RT-qPCR analysis. (A) Validation of microarray data. Four miRNAs were randomly selected to confirm the microarray data. After normalization to U6 level, data are presented as mean \pm SD and obtained average value for each miRNA was used for statistics. "P $<0.05$ compared with wild Mycobacterium tuberculosis (Mtb) group. (B) Effects of regulation of miR-29b expression on interferon- $\gamma$ (IFN- $\gamma$ ) mRNA level in RAW264.7 cells. Downregulation of miR-29b significantly increased IFN- $\gamma$ mRNA level. Upregulation of miR-29b failed to obviously reduce expression of IFN- $\gamma$ mRNA, but there was a trend toward decrease in its level. All experiments were performed in triplicate. ${ }^{*} \mathrm{P}<0.05$ was considered statistically significant. NS, no significance.

group, with 63 miRNAs increased and 42 miRNAs decreased in CWD-Mtb group compared with wild Mtb group (Fig. 3B).

Validation of microarray results by $R T-q P C R$. Four deregulated miRNAs, including miR-468-3p, miR-23a-5p, miR-340-3p and miR-29b, were randomly selected to validate the correctness and confidence of microarray data. Results showed that miR-147-3p and miR-29b were increased, while miR-5097 and miR-872-5p were decreased in CWD-Mtb group in comparison with wild Mtb group (Fig. 4A). The data were consistent with the microarray results.

Effects of modulating miR-29b level on IFN- $\gamma$ mRNA expression. Growing literature shows that cellular miRNAs play important roles in host-pathogen interaction by regulating cytokine production (20-22). It has been reported that IFN- $\gamma$ is a direct target of miR-29b $(23,24)$. Our data showed that miR-29b was increased in CWD-Mtb infected RAW264.7 cells with a concomitant decrease in IFN- $\gamma$ level. To further validate the relationship between miR-29b and IFN- $\gamma$ during intracellular CWD-Mtb infection, we studied the effects of regulation of miR-29b expression on IFN- $\gamma$ level in CWD-Mtb infected RAW264.7 cells. Upregulation or downregulation of miR-29b was achieved by transfection of CWD-Mtb infected RAW264.7 cells with miR-29b mimics or miR-29b inhibitors, respectively. Downregulation of miR-29b resulted in significant increase in IFN- $\gamma$ mRNA. Overexpression of miR-29b failed to lead to obvious decrease in IFN- $\gamma$ mRNA, but there was a trend toward reduction (Fig. 4B).

KEGG pathway analysis of deregulated miRNAs. To identify the most significant candidates and investigate cellular function, involved signaling pathways of potential target genes of deregulated miRNAs were analyzed. Results showed that a wide variety of cellular processes were featured significantly in signaling pathways. Potential targets of overexpressed
miRNAs in CWD-Mtb vs. wild Mtb group were mainly involved in $\mathrm{T}$ cell receptor signaling pathway, pathways in cancer, ABC transporters, bacterial invasion of epithelial cells, while potential targets of downregulated miRNAs in CWD-Mtb vs. wild Mtb group were mainly involved in pathways in cancer, MAPK signaling pathway, neurotrophin signaling pathway, regulation of actin cytoskeleton, long-term potentiation (Fig. 5).

\section{Discussion}

There are many unsolved questions of CWD-forms of Mtb concerning their regulatory mechanisms leading to their survival (25). This study demonstrated that, cell viability was significantly reduced at 12 and $24 \mathrm{~h}$ post wild Mtb infection, by contrast, it was obviously decreased only at $24 \mathrm{~h}$ post CWD-Mtb infection, which indicated that CWD-Mtb exerted less inhibitory effect on cell viability at earlier stage than wild Mtb. Macrophage can secrete pro-inflammatory cytokines, such as TNF- $\alpha$ and IFN- $\gamma$, in order to mediate control of infection with Mtb. Different Mtb strains can induce macrophages to produce different pattern of cytokines (26). We determined the effect of intracellular CWD-Mtb infection on cytokine production in macrophages. The standards for selecting IL-1 $\alpha$, IL-1 $\beta$, IL-6, IL-10, TNF- $\alpha$ and IFN- $\gamma$ in the study was that the four cytokines play important roles in TB infection. IL-1 $\alpha$, IL-1 $\beta$, IL-6 and IL-10 lead to macrophage deactivation (27-30), whereas TNF- $\alpha$ and IFN- $\gamma$ are the central mediators of macrophage activation and inhibit intracellular Mtb growth in macrophages $(31,32)$. Our results showed that levels of IL-1 $\beta$ as well as IL- 6 were increased, and IFN- $\gamma$ was decreased, while TNF- $\alpha$ was not altered in CWD-Mtb group compared with those in wild Mtb group.

In general, intricate regulation of various eukaryotic/host genes is crucial in the development of infectious diseases $(33,34)$. Based on the critical role in regulating innate and adaptive 
$\mathbf{A}$

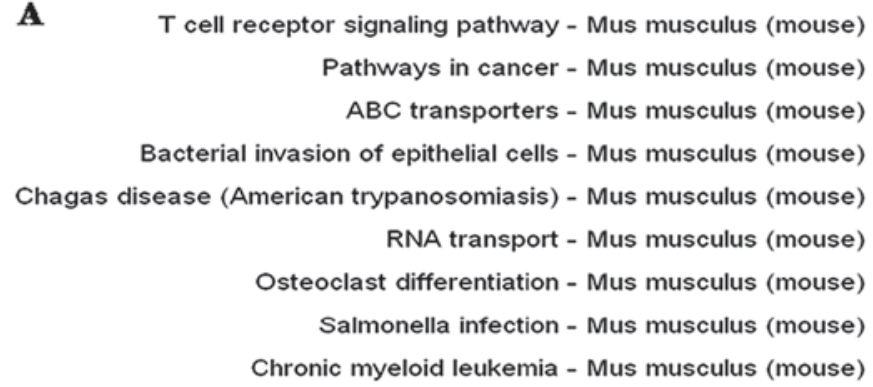

B
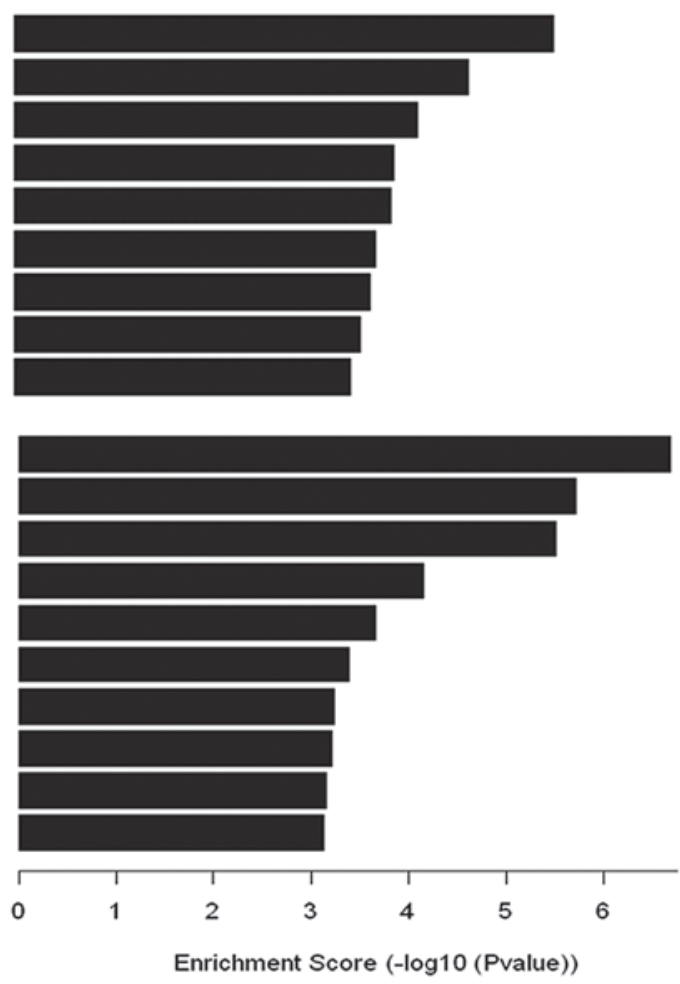

Figure 5. Kyoto Encyclopedia of Genes and Genomes (KEGG) pathway analysis based on (A) upregulated miRNAs and (B) downregulated miRNA targeted genes in cell wall deficient (CWD)-Mtb group vs. wild Mtb group. Enrichment Score value equals $-\log _{10}(\mathrm{P}$-value); $\mathrm{P}<0.05$ was considered statistically significant

immunity, we investigated the effects of intracellular CWD-Mtb infection on miRNA expression in macrophages. Our data showed that miR-147-3p (increased 69-fold) was the most overexpressed miRNA, miR-5097 (decreased 10-fold) was the most downregulated miRNA in CWD-Mtb group compared with wild Mtb group. Among 105 differentially expressed miRNAs, many miRNAs have been found to be associated with diseases: miR$147-3 p$ was deregulated in virus infection (35), miR-5097 was altered in renal cell carcinoma (36), miR-183-5p was involved in ovarian cancer development (37), miR-468-3p was found in association with gastric cancer (38), miR-338-3p participated in the regulation of apoptosis-associated tyrosine kinases and miR93-5p was deregulated in head and neck cancer $(39,40)$. Levels of miRNAs mentioned above were also altered in our study. Previously, the roles of these changed miRNAs in TB infection were not clear, which suggested an additional model of action for these deregulated miRNAs. Several deregulated miRNAs in the study have been reported with involvement in TB infection: miR-191-5p was one of latent TB infection- related miRNAs in macrophages (41); miR-7 and miR-7-5p were upregulated in active TB disease $(15,42)$; miR-720 was increased in latent TB infection group compared with healthy controls. In the study, miR-7b-3p was overexpressed, while miR-191-5p and miR-720 were downregulated in CWD-Mtb group compared with those in wild Mtb group. The data suggested that the above miRNAs may be involved in Mtb-macrophage interactions.

miRNAs play important roles in host-pathogen interaction by regulating cytokine production. Many studies showed that let-7 family members, such as let-7i, let-7g, let-7b, let-7c, and let-7d were deregulated in TB disease $(14,15,43)$. It was reported that let-7 mediated regulation of IL-10 by directly targeting IL-10 $(20,43)$. In the study, compared to control and wild
Mtb group, many let-7 members and IL-10 were deregulated in CWD-Mtb group, which indicated that let-7 members may play important roles in Mtb infection. Our data showed that miR-29b was increased in CWD-Mtb infected cells compared with control as well as wild Mtb infected cells. It was reported that miR-29 was increased in serum, sputum and $\mathrm{CD} 4^{+} \mathrm{T}$ cells from active TB patients $(14,15,44)$, which suggested that miR-29 plays important roles in TB infection. It has been demonstrated that IFN- $\gamma$ is associated with latent TB infection and is a direct target of miR-29b $(23,24,45,46)$. Our data showed that an increased miR-29b level was concomitant with a decreased IFN- $\gamma$ expression in CWD-Mtb group compared with wild Mtb group. To investigate the roles of miR-29b in CWD-Mtb infection, we detected the effects of regulation of miR-29b level on IFN- $\gamma$ expression during intracellular CWD-Mtb infection. Results showed that downregulation of miR-29b resulted in obvious increase in IFN- $\gamma$ level and upregulation of miR-29b failed to decrease IFN- $\gamma$ level, whereas there was a strong trend toward decrease. These data showed that intracellular CWD-Mtb infection could induce distinct immune response by miRNA-mediated gene regulation, which may confer CWD-Mtb an important survival advantage.

Functions of most differentially expressed miRNAs are still largely unknown, such as miR-669e-5p, miR-1839-3p, miR3068-5p, miR-467e-5p and miR-674-3p, but, we can be sure that miRNAs play important roles in the response of macrophages to intracellular CWD-Mtb infection. Based on reported and predicted target genes of the deregulated miRNAs, KEGG pathway analysis was applied to identify enriched pathways involved in intracellular CWD-Mtb infection. Results showed that many signaling pathways were involved in response to CWD-Mtb infection and $\mathrm{T}$ cell receptor signaling pathway, 
MAPK signaling pathway, neurotrophin signaling pathway, regulation of actin cytoskeleton were abundant among the significantly enriched ones.

In conclusion, levels of some cytokines and miRNAs were deregulated in CWD-Mtb infected cells, and intracellular CWD-Mtb infection could elicit different immune response of macrophages by miRNA-mediated gene regulation, which probably determined the differential outcome following different phenotype Mtb infection. These findings open up a new and interesting avenue for an improved understanding of pathogenesis of CWD-Mtb. However, it is difficult to synthesize the results to reach a definitive conclusion based on this single study. Further studies are required to confirm our present findings.

\section{Acknowledgements}

We thank KangChen Biotech for microarray analysis.

\section{Funding}

The study was supported by grants from the 2011 technology innovation frontier exploration key research projects of Weifang Medical University, China (nos. K11TS1004); the Shandong Provincial Natural Science Foundation, China (no. ZR2010HM073) and the National Natural Science Foundation of China (nos. 30972639 and 81170080). The funders had no role in study design, data collection and analysis, decision to publish, or preparation of the manuscript.

\section{Availability of data and material}

The datasets used and/or analyzed during the current study are available from the corresponding author on reasonable request.

\section{Authors' contributions}

ZY and YF participated in bacteria culture, cell infection, microarray and bioinformatics analysis. ZY and KG participated in PCR, modulating analysis and ELISA. RL performed total RNA preparation, quality check and modulating analysis. YF conceived of the study and drafted the manuscript. All authors read and approved the final manuscript.

\section{Ethics approval and consent to participate}

Not applicable.

\section{Consent for publication}

Not applicable.

\section{Competing interests}

The authors declare that they have no competing interests.

\section{Authors' information}

Zhengjun Yi, Department of Laboratory Medicine of Weifang Medical University, Weifang 261053, Shandong Province,
P.R. China; Kunshan Gao, Department of Laboratory Medicine of Weifang Medical University, Weifang 261053, P.R. China; Ruifang Li, Department of Medical Microbiology, Weifang Medical University, Weifang 261053, Shandong Province, P.R. China; Yurong Fu, Department of Medical Microbiology, Weifang Medical University, Weifang 261053, Shandong Province, P.R. China.

\section{References}

1. Markova N, Slavchev G and Michailova L: Unique biological properties of Mycobacterium tuberculosis L-form variants: Impact for survival under stress. Int Microbiol 15: 61-68, 2012.

2. Markova N, Slavchev G and Michailova L: Filterable forms and L-forms of Mycobacterium bovis BCG: Impact for live vaccine features. Hum Vaccin Immunother 8: 759-764, 2012.

3. Michailova L, Kussovski V, Radoucheva T, Jordanova M, Berger W, Rinder H and Markova N: Morphological variability and cell-wall deficiency in Mycobacterium tuberculosis 'heteroresistant' strains. Int J Tuberc Lung Dis 9: 907-914, 2005.

4. Seiler P, Ulrichs T, Bandermann S, Pradl L, Jörg S, Krenn V, Morawietz L, Kaufmann SH and Aichele P: Cell-wall alterations as an attribute of Mycobacterium tuberculosis in latent infection. J Infect Dis 188: 1326-1331, 2003.

5. Koo MS, Subbian S and Kaplan G: Strain specific transcriptional response in Mycobacterium tuberculosis infected macrophages. Cell Commun Signal 10: 2, 2012.

6. Chakraborty P, Kulkarni S, Rajan R and Sainis K: Drug resistant clinical isolates of Mycobacterium tuberculosis from different genotypes exhibit differential host responses in THP-1 cells. PLoS One 8: e62966, 2013.

7. Abuhammad A, Fullam E, Lowe ED, Staunton D, Kawamura A, Westwood IM, Bhakta S, Garner AC, Wilson DL, Seden PT, et al: Piperidinols that show anti-tubercular activity as inhibitors of arylamine N-acetyltransferase: An essential enzyme for mycobacterial survival inside macrophages. PLoS One 7: e52790, 2012.

8. Gautam US, Mehra S, Ahsan MH, Alvarez X, Niu T and Kaushal D: Role of TNF in the altered interaction of dormant Mycobacterium tuberculosis with host macrophages. PLoS One 9: e95220, 2014.

9. Bruns H and Stenger S: New insights into the interaction of Mycobacterium tuberculosis and human macrophages. Future Microbiol 9: 327-341, 2014.

10. van Baal JW, Verbeek RE, Bus P, Fassan M, Souza RF, Rugge M, ten Kate FJ, Vleggaar FP and Siersema PD: microRNA-145 in Barrett's oesophagus: Regulating BMP4 signalling via GATA6. Gut 62: 664-675, 2013.

11. Mohan M, Chandra LC, Torben W, Aye PP, Alvarez X and Lackner AA: miR-190b is markedly upregulated in the intestine in response to simian immunodeficiency virus replication and partly regulates myotubularin-related protein-6 expression. J Immunol 193: 1301-1313, 2014.

12. Roux J, Gonzàlez-Porta $M$ and Robinson-Rechavi $M$ : Comparative analysis of human and mouse expression data illuminates tissue-specific evolutionary patterns of miRNAs. Nucleic Acids Res 40: 5890-5900, 2012.

13. Wu Z, Lu H, Sheng J and Li L: Inductive microRNA-21 impairs anti-mycobacterial responses by targeting IL-12 and Bcl-2. FEBS Lett 586: 2459-2467, 2012.

14. Ma C, Li Y, Li M, Deng G, Wu X, Zeng J, Hao X, Wang X, Liu J, Cho WC, et al: microRNA-124 negatively regulates TLR signaling in alveolar macrophages in response to mycobacterial infection. Mol Immunol 62: 150-158, 2014.

15. Dorhoi A, Iannaccone M, Farinacci M, Faé KC, Schreiber J, Moura-Alves P, Nouailles G, Mollenkopf HJ, Oberbeck-Müller D, Jörg S, et al: MicroRNA-223 controls susceptibility to tuberculosis by regulating lung neutrophil recruitment. J Clin Invest 123: 4836-4848, 2013.

16. Kumar R, Halder P, Sahu SK, Kumar M, Kumari M, Jana K, Ghosh Z, Sharma P, Kundu M and Basu J: Identification of a novel role of ESAT-6-dependent miR-155 induction during infection of macrophages with Mycobacterium tuberculosis. Cell Microbiol 14: 1620-1631, 2012.

17. Sutherland JS, Adetifa IM, Hill PC, Adegbola RA and Ota MO: Pattern and diversity of cytokine production differentiates between Mycobacterium tuberculosis infection and disease. Eur J Immunol 39: 723-729, 2009. 
18. Sharma S and Bose M: Role of cytokines in immune response to pulmonary tuberculosis. Asian Pac J Allergy Immunol 19: 213-219, 2001

19. Singh PP and Goyal A: Interleukin-6: A potent biomarker of mycobacterial infection. Springerplus 2: 686, 2013.

20. Swaminathan S, Suzuki K, Seddiki N, Kaplan W, Cowley MJ, Hood CL, Clancy JL, Murray DD, Méndez C, Gelgor L, et al: Differential regulation of the Let-7 family of microRNAs in $\mathrm{CD}^{+} \mathrm{T}$ cells alters IL-10 expression. J Immunol 188: 6238-6246, 2012.

21. Huang HC, Yu HR, Huang LT, Huang HC, Chen RF, Lin IC, Ou CY, Hsu TY and Yang KD: miRNA-125b regulates TNF- $\alpha$ production in $\mathrm{CD}_{14}{ }^{+}$neonatal monocytes via post-transcriptional regulation. J Leukoc Biol 92: 171-182, 2012.

22. Sarma NJ, Tiriveedhi V, Crippin JS, Chapman WC and Mohanakumar T: Hepatitis C virus-induced changes in microRNA 107 (miRNA-107) and miRNA-449a modulate CCL2 by targeting the interleukin-6 receptor complex in hepatitis. J Virol 88: 3733-3743, 2014.

23. Ma F, Xu S, Liu X, Zhang Q, Xu X, Liu M, Hua M, Li N, Yao H and Cao X: The microRNA miR-29 controls innate and adaptive immune responses to intracellular bacterial infection by targeting interferon- $\gamma$. Nat Immunol 12: 861-869, 2011.

24. Steiner DF, Thomas MF, Hu JK, Yang Z, Babiarz JE, Allen CD, Matloubian M, Blelloch R and Ansel KM: MicroRNA-29 regulates T-box transcription factors and interferon- $\gamma$ production in helper T cells. Immunity 35: 169-181, 2011.

25. Almenoff PL, Johnson A, Lesser M and Mattman LH: Growth of acid fast $\mathrm{L}$ forms from the blood of patients with sarcoidosis. Thorax 51: 530-533, 1996.

26. Chen YY, Chang JR, Huang WF, Hsu SC, Kuo SC, Sun JR and Dou HY: The pattern of cytokine production in vitro induced by ancient and modern Beijing Mycobacterium tuberculosis strains. PLoS One 9: e94296, 2014.

27. Briken V, Ahlbrand SE and Shah S: Mycobacterium tuberculosis and the host cell inflammasome: A complex relationship. Front Cell Infect Microbiol 3: 62, 2013.

28. Tang S, Cui H, Yao L, Hao X, Shen Y, Fan L, Sun H, Zhang Z and Huang JA: Increased cytokines response in patients with tuberculosis complicated with chronic obstructive pulmonary disease. PLoS One 8: e62385, 2013.

29. Yang R, Xi C, Sita DR, Sakai S, Tsuchiya K, Hara H, Shen Y, Qu H, Fang R, Mitsuyama M, et al: The RD1 locus in the Mycobacterium tuberculosis genome contributes to the maturation and secretion of IL-1 $\alpha$ from infected macrophages through the elevation of cytoplasmic calcium levels and calpain activation. Pathog Dis 70: 51-60, 2014.

30. Das S, Banerjee S, Majumder S, Chowdhury BP, Goswami A, Halder K, Chakraborty U, Pal NK and Majumdar S: Immune subversion by Mycobacterium tuberculosis through CCR 5 mediated signaling: Involvement of IL-10. PLoS One 9: e92477, 2014.

31. Denis $M$ and Gregg EO: Recombinant tumour necrosis factoralpha decreases whereas recombinant interleukin-6 increases growth of a virulent strain of Mycobacterium avium in human macrophages. Immunology 71: 139-141, 1990.

32. Cavalcanti YV, Brelaz MC, Neves JK, Ferraz JC and Pereira VR Role of TNF-alpha, IFN-gamma, and IL-10 in the development of pulmonary tuberculosis. Pulm Med 2012: 745483, 2012.

33. Chen CZ, Schaffert S, Fragoso R and Loh C: Regulation of immune responses and tolerance: The microRNA perspective. Immunol Rev 253: 112-128, 2013.
34. Foster PS, Plank M, Collison A, Tay HL, Kaiko GE, Li J, Johnston SL, Hansbro PM, Kumar RK, Yang M, et al: The emerging role of microRNAs in regulating immune and inflammatory responses in the lung. Immunol Rev 253: 198-215, 2013.

35. A Hicks J, Yoo D and Liu HC: Characterization of the microRNAome in porcine reproductive and respiratory syndrome virus infected macrophages. PLoS One 8: e82054, 2013.

36. Birkhäuser FD, Koya RC, Neufeld C, Rampersaud EN, Lu X, Micewicz ED, Chodon T, Atefi M, Kroeger N, Chandramouli GV, et al: Dendritic cell-based immunotherapy in prevention and treatment of renal cell carcinoma: Efficacy, safety, and activity of Ad-GM.CAIX in immunocompetent mouse models. J Immunother 36: 102-111, 2013.

37. Wang L, Zhu MJ, Ren AM, Wu HF, Han WM, Tan RY and Tu RQ: A ten-microRNA signature identified from a genomewide microRNA expression profiling in human epithelial ovarian cancer. PLoS One 9: e96472, 2014.

38. Su Y, Ni Z, Wang G, Cui J, Wei C, Wang J, Yang Q, Xu Y and Li F: Aberrant expression of microRNAs in gastric cancer and biological significance of miR-574-3p. Int Immunopharmacol 13: 468-475, 2012.

39. Kos A, Olde Loohuis NF, Wieczorek ML, Glennon JC, Martens GJ, Kolk SM and Aschrafi A: A potential regulatory role for intronic microRNA-338-3p for its host gene encoding apoptosis-associated tyrosine kinase. PLoS One 7: e31022, 2012.

40. Summerer I, Niyazi M, Unger K, Pitea A, Zangen V, Hess J, Atkinson MJ, Belka C, Moertl S and Zitzelsberger H: Changes in circulating microRNAs after radiochemotherapy in head and neck cancer patients. Radiat Oncol 8: 296, 2013.

41. Meng QL, Liu F, Yang XY, Liu XM, Zhang X, Zhang C and Zhang ZD: Identification of latent tuberculosis infectionrelated microRNAs in human U937 macrophages expressing Mycobacterium tuberculosis Hsp16.3. BMC Microbiol 14: 37, 2014.

42. Fu Y, Yi Z, Li J and Li R: Deregulated microRNAs in CD4 ${ }^{+}$ $T$ cells from individuals with latent tuberculosis versus active tuberculosis. J Cell Mol Med 18: 503-513, 2014.

43. Jiang L, Cheng Z, Qiu S, Que Z, Bao W, Jiang C, Zou F, Liu P and Liu J: Altered let-7 expression in Myasthenia gravis and let-7c mediated regulation of IL-10 by directly targeting IL-10 in Jurkat cells. Int Immunopharmacol 14: 217-223, 2012.

44. Kleinsteuber K, Heesch K, Schattling S, Kohns M, Sander-Jülch C, Walzl G, Hesseling A, Mayatepek E, Fleischer B, Marx FM, et al: Decreased expression of miR-21, miR-26a, miR-29a, and miR$142-3 p$ in $\mathrm{CD}^{+} \mathrm{T}$ cells and peripheral blood from tuberculosis patients. PLoS One 8: e61609, 2013.

45. Masood KI, Hussain R, Rao N, Rottenberg ME, Salahuddin N, Irfan $M$ and Hasan Z: Differential Early Secreted Antigen Target (ESAT) $6 \mathrm{kDa}$-induced IFN- $\gamma$ and SOCS1 expression distinguishes latent and active tuberculosis. J Infect Dev Ctries 8: 59-66, 2014.

46. Adetifa IM, Ota MO, Jeffries DJ, Lugos MD, Hammond AS, Battersby NJ, Owiafe PK, Donkor SD, Antonio M, Ibanga HB, et al: Interferon- $\gamma$ ELISPOT as a biomarker of treatment efficacy in latent tuberculosis infection: A clinical trial. Am J Respir Crit Care Med 187: 439-445, 2013. 